\section{Cahiers de Narratologie}

Analyse et théorie narratives

$35 \mid 2019$

Le style comme événement

\title{
Stefano Bragato, Futurismo in nota. Studio sui taccuini di Marinetti
}

\section{Barbara Meazzi}

\section{(2) OpenEdition \\ 12 Journals}

Electronic version

URL: http://journals.openedition.org/narratologie/9248

DOI: $10.4000 /$ narratologie.9248

ISSN: 1765-307X

Publisher

LIRCES

\section{Electronic reference}

Barbara MeazZi, « STEFANo BRAgATO, FUTURISMO IN NOTA. STUdIO SUI TACCUINI DI MARINETTI 》, Cahiers de Narratologie [Online], 35 | 2019, Online since 03 September 2019, connection on 24 September 2020. URL : http://journals.openedition.org/narratologie/9248 ; DOI : https://doi.org/ 10.4000/narratologie.9248

This text was automatically generated on 24 September 2020.

Article L.111-1 du Code de la propriété intellectuelle. 


\title{
Stefano Bragato, Futurismo in nota. Studio sui taccuini di Marinetti
}

\author{
Barbara Meazzi
}

\section{REFERENCES}

Stefano Bragato, Futurismo in nota. Studio sui taccuini di Marinetti, Florence, Franco Cesati Editore, 2018

1 Peu de choses ont été écrites sur le journal de F.T. Marinetti, fondateur et animateur de l'avant-garde futuriste, avant et depuis sa publication partielle en 1987, sans doute parce qu'on a pu à l'époque considérer l'édition des Taccuini d'Alberto Bertoni comme définitive. Parue à Bologne, celle-ci restituait généreusement une partie importante de la vie quotidienne du fondateur du futurisme, et tout particulièrement celle des années de la Grande guerre. L'ouvrage rendait un grand service à la communauté des chercheurs et des doctorants travaillant sur le futurisme et, plus largement, sur la Première Guerre mondiale côté italien. Par ailleurs, sa densité était telle (il s'agissait de plusieurs centaines de pages, transcrites à partir de notes manuscrites de Marinetti entre 1915 et 1926) qu'il avait fallu plusieurs années pour les appréhender. Aucune raison ne poussait les chercheurs à douter de la fiabilité d'un corpus aussi vaste, enfin mis à la disposition du public : pourtant, Bertoni lui-même avait alerté ses lecteurs, en introduction, reconnaissant une transcription parfois incomplète et très soucieuse, affirmait-il, du droit au respect de la vie privée de la famille Marinetti.

2 Ce n'est donc que bien des années plus tard que l'on s'est interrogé sur la consistance de cette transcription, alors que certains chercheurs avaient par ailleurs transcrit des extraits inédits du journal manuscrit marinettien ${ }^{1}$ : de même, lorsqu'on a pu travailler sur les archives du futurisme pour corriger certaines erreurs factuelles, on s'est également interrogé sur la nature et sur l'importance des omissions de l'édition de Bertoni $^{2}$. Si les Taccuini contenaient certaines ébauches de manifestes et de textes 
romanesques - comme le roman L'alcova d'acciaio - les manuscrits originaux devaient receler bien d'autres matériaux pouvant ouvrir des perspectives nouvelles quant à la conception des récits du futurisme.

3 L'ouvrage que vient de publier Stefano Bragato, issu de sa thèse de doctorat, porte précisément sur les nouvelles perspectives offertes par une différente appréhension du journal de Marinetti, lu ici avec un souci philologique inédit. Le seul grief que l'on pourrait lui faire, autant l'annoncer d'emblée, serait qu'il tient peu compte de la récente et abondante bibliographie en français ou en espagnol consacrée au futurisme. Un exemple entre tous: sur l'image de la femme, il aurait probablement été plus pertinent de se référer à l'essai de Silvia Contarini de 2006 qu'à celui de 1996 de Cinzia Sartini Blum ${ }^{3}$, tout aussi important mais plus daté; mais mis à part ces quelques références bibliographiques manquantes, Bragato livre un travail remarquable et fort bien écrit.

4 Plutôt que de considérer le journal de Marinetti comme un "diario di vita ", une chronique de vie quotidienne, l'auteur suggère d'appréhender le manuscrit comme un "instrument de travail poétique ", le lieu où la réalité vécue est transfigurée en littérature. L'épopée futuriste, en somme, trouverait son point de départ dans l'écriture quotidienne de Marinetti analysée avec une rigueur philologique exemplaire : de plus, face à la difficulté de manier seul un corpus démesuré (Marinetti aurait rédigé son journal sur plus de dix ans), le chercheur a choisi d'articuler son travail autour de trois moments, autant d'échantillons lui permettant de mener à bien son enquête : l'exorde de Marinetti soldat, en 1915 ; la composition de L'alcova d'acciaio en 1921 ; le voyage au Brésil en 1926. Ainsi, plutôt que de retranscrire la totalité du corpus, Bragato présente les extraits saillants sans perdre de vue le fait qu'il s'agit de récits conçus comme des pré-textes, des matériaux abrupts de création poétique.

5 Bragato suit dès lors Marinetti au front, et compare les pages des Taccuini aux textes publiés aussi bien dans la Gazzetta dello Sport que dans des planches motslibristes, et croise ensuite ces documents avec les notes que rédigent, chacun de leur côté, les peintres futuristes Umberto Boccioni et Anselmo Bucci. Outre la transfiguration poétique de l'écriture de Marinetti, sont ainsi mises en évidence quelques distorsions des évènements que Bragato définit comme une «chronologie créative»: Marinetti a parfois pu inventer une guerre qui n'a pas eu lieu. Par exemple, il semble qu'il n'ait pas participé avec Boccioni à l'assaut de Dosso Casina du 24 au 26 octobre 1915, alors même qu'il publie une planche de mots en liberté intitulée justement «Con Boccioni a Dosso Casina $»^{4}$, avec d'autres articles célébrant la vaillance des soldats futuristes en cette occasion. Force est de constater que le journal de guerre de Marinetti se différencie ainsi des journaux de guerre de ses contemporains (Piero Jahier, Renato Serra, ou encore Carlo Emilio Gadda).

L'analyse de Bragato se focalise ensuite, dans sa deuxième partie, sur les années 1918-1921, et prend appui sur le roman L'alcova d'acciaio, publié au mois d'avril 1921 chez l'éditeur milanais Vitagliano, où Marinetti raconte sa vie au front entre les mois de juin et novembre 1918. Le roman est élaboré à partir du journal mais, contrairement à la période 1915-1916, où la représentation de la guerre évoquait la présence de ses compagnons futuristes, en 1918-1921 Marinetti renonce à décrire sa vie au front comme une "expérience collective du groupe futuriste " et se met en scène tout seul, avec sa chienne. Les faits et gestes de l'armée italienne sont célébrés à maintes reprises dans le roman, mais la guerre est surtout une question personnelle, abordée et 
juxtaposée à la matière amoureuse : les exploits de Marinetti-Casanova sous-tendent en fait l'amour - toujours impétueux - pour la patrie.

7 Bragato rappelle judicieusement qu'avec L'alcova d'acciaio Marinetti entend modifier la perception du futurisme, notamment auprès du grand public, et souhaite, de toute évidence, en faire autre chose qu'un mouvement artistique réservé à une élite, surtout après avoir été échaudé par le rapprochement avec Mussolini et le fascisme. L'analyse philologique de la transformation textuelle demeure très subtile et toujours rigoureuse, mais certaines interrogations quant à la genèse du roman restent ouvertes : dans sa première version, L'alcova d'acciaio aurait été dictée au journaliste Enrico Santamaria, mais si l'on s'en tient à la correspondance de Benedetta (qui épousera Marinetti en 1926), c'est à elle que Marinetti aurait dicté la version définitive du roman ${ }^{5}$. Sans doute conviendra-t-il de revenir sur cette question, alors que l'hypothèse initiale de Stefano Bragato informe également dans la troisième partie de l'ouvrage, consacrée à l'analyse du voyage au Brésil de 1926.

8 En effet, le choix du corpus pour cette dernière partie nous rappelle que le travail de Bragato porte sur l'hypothèse du journal comme laboratoire d'écriture créative. Le chercheur s'intéresse ici à des nouvelles moins connues de Marinetti, dont l'analyse attentive permet de conclure que le fondateur du futurisme était très sensible à ce qu'aujourd'hui on appellerait les transferts culturels, et qu'il était fasciné par l'idée que la société européenne puisse s'enrichir au contact avec l'art primitif (et pas le contraire). Bragato souligne à raison que, contrairement à bien d'autres artistes contemporains, Marinetti ne s'intéresse pas aux cultures «des Suds» en ce qu'elles représentent des alternatives édéniques, mais parce qu'il ressent toujours très fort en lui l'attrait pour le "sauvage ", une composante primitive constitutive de son essence et donc de l'essence même du futurisme : Marinetti était né en Alexandrie d'Égypte et y avait vécu pendant toute son adolescence. Bragato rappelle, à juste titre, l'importance de cet aspect que l'on perçoit tout particulièrement dans Mafarka le Futuriste (1910), et il insiste sur la persistance de cette dimension pouvant, selon Marinetti, revitaliser la civilisation occidentale toute entière.

Il faudra sans doute revenir sur les questions soulevées par cet ouvrage, notamment dans cette troisième partie, fort originale. La voie ouverte par Stefano Bragato nous conforte dans l'idée qu'une relecture des œuvres de Marinetti et du futurisme est indispensable et qu'il est grand temps de repenser le futurisme en repartant des textes de création : il faut lui en être reconnaissant.

\section{NOTES}

1. Lawrence Rainey, Laura Wittman, «Selections from the Unpublished Diaries of F.T. Marinetti: Introduction and Notes by ", in Modernism/modernity, I (1993), 3, p. 1-44.

2. Cf. Barbara Meazzi, «Les projets romanesques de Marinetti: (im)précis de génétique textuelle", in Cahiers de Narratologie, $n^{\circ} 24$, Nice, septembre 2013, http:// narratologie.revues.org/, ainsi que la thèse de doctorat de Simona Fava, en cours de rédaction. 
3. Silvia Contarini, Mythes, modèles et représentations de la femme dans la théorie et la littérature futuriste, Nanterre, Publidix-Presses Universitaires de Paris 10, 2006 ; Cinzia Sartini Blum, The Other Modernism. F. T. Marinetti's Futurist Fiction of Power, Berkeley, University of California Press, 1996.

4. F.T. Marinetti, « Con Boccioni a Dosso Casina », in L'Italia futurista, I, 25 août 1916.

5. La lettre en question, non datée et écrite depuis Oneglia, où Benedetta a vécu au début de sa relation avec Marinetti en attendant que celui-ci prépare l'appartement de Milan, fait partie du Fonds Benedetta Cappa Marinetti conservé au Paul Getty Research Institute. 